\title{
On certain differential sandwich theorems involving a generalized Sălăgean operator and Ruscheweyh operator
}

\author{
Andrei Loriana
}

\begin{abstract}
In the present paper we introduce sufficient conditions for subordination and superordination involving the operator $D R_{\lambda}^{m, n}$ and also we obtain sandwich-type results.
\end{abstract}

\section{Introduction}

Let $\mathcal{H}(U)$ be the class of analytic function in the open unit disc of the complex plane $U=\{z \in \mathbb{C}:|z|<1\}$. Let $\mathcal{H}(a, n)$ be the subclass of $\mathcal{H}(U)$ consisting of functions of the form $f(z)=a+a_{n} z^{n}+a_{n+1} z^{n+1}+\ldots$.

Let $\mathcal{A}_{n}=\left\{f \in \mathcal{H}(U): f(z)=z+a_{n+1} z^{n+1}+\ldots, z \in U\right\}$ and $\mathcal{A}=\mathcal{A}_{1}$.

Denote by $K=\left\{f \in \mathcal{A}: \operatorname{Re} \frac{z f^{\prime \prime}(z)}{f^{\prime}(z)}+1>0, z \in U\right\}$, the class of normalized convex functions in $U$.

Let the functions $f$ and $g$ be analytic in $U$. We say that the function $f$ is subordinate to $g$, written $f \prec g$, if there exists a Schwarz function $w$, analytic in $U$, with $w(0)=0$ and $|w(z)|<1$, for all $z \in U$, such that $f(z)=g(w(z))$, for all $z \in U$. In particular, if the function $g$ is univalent in $U$, the above subordination is equivalent to $f(0)=g(0)$ and $f(U) \subset g(U)$.

Key Words: analytic functions, differential operator, differential subordination, differential superordination.

2010 Mathematics Subject Classification: Primary 30C45.

Received: 5 May, 2014.

Revised: 20 June, 2014.

Accepted: 29 June, 2014. 
ON CERTAIN DIFFERENTIAL SANDWICH THEOREMS INVOLVING A

Let $\psi: \mathbb{C}^{3} \times U \rightarrow \mathbb{C}$ and $h$ be an univalent function in $U$. If $p$ is analytic in $U$ and satisfies the second order differential subordination

$$
\psi\left(p(z), z p^{\prime}(z), z^{2} p^{\prime \prime}(z) ; z\right) \prec h(z), \quad \text { for } z \in U,
$$

then $p$ is called a solution of the differential subordination. The univalent function $q$ is called a dominant of the solutions of the differential subordination, or more simply a dominant, if $p \prec q$ for all $p$ satisfying (1). A dominant $\widetilde{q}$ that satisfies $\widetilde{q} \prec q$ for all dominants $q$ of (1) is said to be the best dominant of (1). The best dominant is unique up to a rotation of $U$.

Let $\psi: \mathbb{C}^{2} \times U \rightarrow \mathbb{C}$ and $h$ analytic in $U$. If $p$ and $\psi\left(p(z), z p^{\prime}(z), z^{2} p^{\prime \prime}(z) ; z\right)$ are univalent and if $p$ satisfies the second order differential superordination

$$
h(z) \prec \psi\left(p(z), z p^{\prime}(z), z^{2} p^{\prime \prime}(z) ; z\right), \quad z \in U,
$$

then $p$ is a solution of the differential superordination (2) (if $f$ is subordinate to $F$, then $F$ is called to be superordinate to $f$ ). An analytic function $q$ is called a subordinant if $q \prec p$ for all $p$ satisfying (2). An univalent subordinant $\widetilde{q}$ that satisfies $q \prec \widetilde{q}$ for all subordinants $q$ of (2) is said to be the best subordinant.

Miller and Mocanu [17] obtained conditions $h, q$ and $\psi$ for which the following implication holds

$$
h(z) \prec \psi\left(p(z), z p^{\prime}(z), z^{2} p^{\prime \prime}(z) ; z\right) \Rightarrow q(z) \prec p(z) .
$$

For two functions $f(z)=z+\sum_{j=2}^{\infty} a_{j} z^{j}$ and $g(z)=z+\sum_{j=2}^{\infty} b_{j} z^{j}$ analytic in the open unit disc $U$, the Hadamard product (or convolution product) of $f(z)$ and $g(z)$, written as $(f * g)(z)$, is defined by

$$
f(z) * g(z)=(f * g)(z)=z+\sum_{j=2}^{\infty} a_{j} b_{j} z^{j} .
$$

Definition 1. (Al Oboudi [7]) For $f \in \mathcal{A}, \lambda \geq 0$ and $n \in \mathbb{N}$, the operator $D_{\lambda}^{m}$ is defined by $D_{\lambda}^{m}: \mathcal{A} \rightarrow \mathcal{A}$,

$$
\begin{aligned}
D_{\lambda}^{0} f(z)= & f(z) \\
D_{\lambda}^{1} f(z)= & (1-\lambda) f(z)+\lambda z f^{\prime}(z)=D_{\lambda} f(z) \\
& \cdots \\
D_{\lambda}^{m} f(z)= & (1-\lambda) D_{\lambda}^{m-1} f(z)+\lambda z\left(D_{\lambda}^{m} f(z)\right)^{\prime}=D_{\lambda}\left(D_{\lambda}^{m-1} f(z)\right), \text { for } z \in U .
\end{aligned}
$$

Remark 1. If $f \in \mathcal{A}$ and $f(z)=z+\sum_{j=2}^{\infty} a_{j} z^{j}$, then $D_{\lambda}^{m} f(z)=z+$ $\sum_{j=2}^{\infty}[1+(j-1) \lambda]^{m} a_{j} z^{j}$, for $z \in U$.

Remark 2. For $\lambda=1$ in the above definition we obtain the Sălăgean differential operator [20]. 
ON CERTAIN DIFFERENTIAL SANDWICH THEOREMS INVOLVING A

Definition 2. (Ruscheweyh [19]) For $f \in \mathcal{A}$ and $n \in \mathbb{N}$, the operator $R^{n}$ is defined by $R^{n}: \mathcal{A} \rightarrow \mathcal{A}$,

$$
\begin{aligned}
R^{0} f(z)= & f(z) \\
R^{1} f(z)= & z f^{\prime}(z) \\
& \cdots \\
(n+1) R^{n+1} f(z)= & z\left(R^{n} f(z)\right)^{\prime}+n R^{n} f(z), \quad z \in U .
\end{aligned}
$$

Remark 3. If $f \in \mathcal{A}, f(z)=z+\sum_{j=2}^{\infty} a_{j} z^{j}$, then

$$
R^{n} f(z)=z+\sum_{j=2}^{\infty} \frac{(n+j-1) !}{n !(j-1) !} a_{j} z^{j} \text { for } z \in U
$$

Using the results of Miller and Mocanu [17], [18], Bulboaca [14] considered certain classes of first order differential superordinations. Recently, many authors [15], [22], [21], [16], [23], have used the results of Bulboaca and obtain certain sufficient conditions applying first order differential subordinations and superordinations. In order to prove our subordination and superordination results, we make use of the following:

Definition 3. [18] Denote by $Q$ the set of all functions $f$ that are analytic and injective on $\bar{U} \backslash E(f)$, where $E(f)=\left\{\zeta \in \partial U: \lim _{z \rightarrow \zeta} f(z)=\infty\right\}$, and are such that $f^{\prime}(\zeta) \neq 0$ for $\zeta \in \partial U \backslash E(f)$.

Lemma 1. [18] Let the function $q$ be univalent in the unit disc $U$ and $\theta$ and $\phi$ be analytic in a domain $D$ containing $q(U)$ with $\phi(w) \neq 0$ when $w \in q(U)$. Set $Q(z)=z q^{\prime}(z) \phi(q(z))$ and $h(z)=\theta(q(z))+Q(z)$. Suppose that

1. $Q$ is starlike univalent in $U$ and

2. $\operatorname{Re}\left(\frac{z h^{\prime}(z)}{Q(z)}\right)>0$ for $z \in U$.

If $p$ is analytic with $p(0)=q(0), p(U) \subseteq D$ and

$$
\theta(p(z))+z p^{\prime}(z) \phi(p(z)) \prec \theta(q(z))+z q^{\prime}(z) \phi(q(z)),
$$

then $p(z) \prec q(z)$ and $q$ is the best dominant.

Lemma 2. [14] Let the function $q$ be convex univalent in the open unit disc $U$ and $\nu$ and $\phi$ be analytic in a domain $D$ containing $q(U)$. Suppose that

1. $\operatorname{Re}\left(\frac{\nu^{\prime}(q(z))}{\phi(q(z))}\right)>0$ for $z \in U$ and

2. $\psi(z)=z q^{\prime}(z) \phi(q(z))$ is starlike univalent in $U$. 
ON CERTAIN DIFFERENTIAL SANDWICH THEOREMS INVOLVING A

If $p(z) \in \mathcal{H}[q(0), 1] \cap Q$, with $p(U) \subseteq D$ and $\nu(p(z))+z p^{\prime}(z) \phi(p(z))$ is univalent in $U$ and

$$
\nu(q(z))+z q^{\prime}(z) \phi(q(z)) \prec \nu(p(z))+z p^{\prime}(z) \phi(p(z)),
$$

then $q(z) \prec p(z)$ and $q$ is the best subordinant.

\section{Main results}

Definition 4. Let $\lambda \geq 0$ and $n, m \in \mathbb{N}$. Denote by $D R_{\lambda}^{m, n}: \mathcal{A} \rightarrow \mathcal{A}$ the operator given by the Hadamard product of the generalized Sălăgean operator $D_{\lambda}^{m}$ and the Ruscheweyh operator $R^{n}$,

$$
D R_{\lambda}^{m, n} f(z)=\left(D_{\lambda}^{m} * R^{n}\right) f(z),
$$

for any $z \in U$ and each nonnegative integers $m, n$.

Remark 4. If $f \in \mathcal{A}$ and $f(z)=z+\sum_{j=2}^{\infty} a_{j} z^{j}$, then

$D R_{\lambda}^{m, n} f(z)=z+\sum_{j=2}^{\infty}[1+(j-1) \lambda]^{m} \frac{(n+j-1) !}{n !(j-1) !} a_{j}^{2} z^{j}$, for $z \in U$.

This operator was studied in [12] and [13].

Remark 5. For $\lambda=1, m=n$, we obtain the Hadamard product $S R^{n}$ [1] of the Sălăgean operator $S^{n}$ and Ruscheweyh derivative $R^{n}$, which was studied in [2], [3].

Remark 6. For $m=n$ we obtain the Hadamard product $D R_{\lambda}^{n}$ [4] of the generalized Sălăgean operator $D_{\lambda}^{n}$ and Ruscheweyh derivative $R^{n}$, which was studied in [5], [6], [8], [9], [10], [11].

Using simple computation one obtains the next result.

Proposition 1. [12].For $m, n \in \mathbb{N}$ and $\lambda \geq 0$ we have

$$
D R_{\lambda}^{m+1, n} f(z)=(1-\lambda) D R_{\lambda}^{m, n} f(z)+\lambda z\left(D R_{\lambda}^{m, n} f(z)\right)^{\prime}
$$

and

$$
z\left(D R_{\lambda}^{m, n} f(z)\right)^{\prime}=(n+1) D R_{\lambda}^{m, n+1} f(z)-n D R_{\lambda}^{m, n} f(z) .
$$

First, our purpose is to find sufficient conditions for certain normalized analytic functions $f$ such that

$$
q_{1}(z) \prec \frac{z^{\delta} D R_{\lambda}^{m, n+1} f(z)}{\left(D R_{\lambda}^{m, n} f(z)\right)^{1+\delta}} \prec q_{2}(z), \quad z \in U, 0<\delta \leq 1
$$

where $q_{1}$ and $q_{2}$ are given univalent functions. 
ON CERTAIN DIFFERENTIAL SANDWICH THEOREMS INVOLVING A

Theorem 1. Let $\frac{z^{\delta} D R_{\lambda}^{m, n+1} f(z)}{\left(D R_{\lambda}^{m, n} f(z)\right)^{1+\delta}} \in \mathcal{H}(U), z \in U, f \in \mathcal{A}, m, n \in \mathbb{N}, \lambda \geq 0$, $0<\delta \leq 1$ and let the function $q(z)$ be convex and univalent in $U$ such that $q(0)=1$. Assume that

$$
\operatorname{Re}\left(1+\frac{\alpha}{\beta} q(z)-\frac{z q^{\prime}(z)}{q(z)}+\frac{z q^{\prime \prime}(z)}{q^{\prime}(z)}\right)>0, \quad z \in U,
$$

for $\alpha, \beta \in \mathbb{C}, \beta \neq 0, z \in U$, and

$$
\begin{gathered}
\psi_{\lambda}^{m, n}(\alpha, \beta, \delta ; z):=\alpha \frac{z^{\delta} D R_{\lambda}^{m, n+1} f(z)}{\left(D R_{\lambda}^{m, n} f(z)\right)^{1+\delta}}+ \\
+\beta\left[\delta(n+1)-1+(n+2) \frac{D R_{\lambda}^{m, n+2} f(z)}{D R_{\lambda}^{m, n+1} f(z)}-(1+\delta)(n+1) \frac{D R_{\lambda}^{m, n+1} f(z)}{D R_{\lambda}^{m, n} f(z)}\right] .
\end{gathered}
$$

If $q$ satisfies the following subordination

$$
\psi_{\lambda}^{m, n}(\alpha, \beta, \delta ; z) \prec \alpha q(z)+\frac{\beta z q^{\prime}(z)}{q(z)},
$$

for $\alpha, \beta \in \mathbb{C}, \beta \neq 0$ then

$$
\frac{z^{\delta} D R_{\lambda}^{m, n+1} f(z)}{\left(D R_{\lambda}^{m, n} f(z)\right)^{1+\delta}} \prec q(z), \quad z \in U
$$

and $q$ is the best dominant.

Proof. Let the function $p$ be defined by $p(z):=\frac{z^{\delta} D R_{\lambda}^{m, n+1} f(z)}{\left(D R_{\lambda}^{m, n} f(z)\right)^{1+\delta}}, z \in U, z \neq 0$, $0<\delta \leq 1, f \in \mathcal{A}$. The function $p$ is analytic in $U$ and $p(0)=1$.

Differentiating this function, with respect to $z$, we get

$$
z p^{\prime}(z)=\frac{z^{\delta} D R_{\lambda}^{m, n+1} f(z)}{\left(D R_{\lambda}^{m, n} f(z)\right)^{1+\delta}}\left[\delta+\frac{z\left(D R_{\lambda}^{m, n+1} f(z)\right)^{\prime}}{D R_{\lambda}^{m, n+1} f(z)}-(1+\delta) \frac{z\left(D R_{\lambda}^{m, n} f(z)\right)^{\prime}}{D R_{\lambda}^{m, n} f(z)}\right] .
$$

By using the identity (4), we obtain

$\frac{z p^{\prime}(z)}{p(z)}=\delta(n+1)-1+(n+2) \frac{D R_{\lambda}^{m, n+2} f(z)}{D R_{\lambda}^{m, n+1} f(z)}-(1+\delta)(n+1) \frac{D R_{\lambda}^{m, n+1} f(z)}{D R_{\lambda}^{m, n} f(z)}$.

By setting $\theta(w):=\alpha w$ and $\phi(w):=\frac{\beta}{w}, \alpha, \beta \in \mathbb{C}, \beta \neq 0$ it can be easily verified that $\theta$ is analytic in $\mathbb{C}, \phi$ is analytic in $\mathbb{C} \backslash\{0\}$ and that $\phi(w) \neq 0$, $w \in \mathbb{C} \backslash\{0\}$.

Also, by letting $Q(z)=z q^{\prime}(z) \phi(q(z))=\frac{\beta z q^{\prime}(z)}{q(z)}$,we find that $Q(z)$ is starlike univalent in $U$. 
ON CERTAIN DIFFERENTIAL SANDWICH THEOREMS INVOLVING A

Let $h(z)=\theta(q(z))+Q(z)=\alpha q(z)+\frac{\beta z q^{\prime}(z)}{q(z)}, z \in U$.

If we derive the function $Q$, with respect to $z$, perform calculations, we have $\operatorname{Re}\left(\frac{z h^{\prime}(z)}{Q(z)}\right)=\operatorname{Re}\left(1+\frac{\alpha}{\beta} q(z)-\frac{z q^{\prime}(z)}{q(z)}+\frac{z q^{\prime \prime}(z)}{q^{\prime}(z)}\right)>0$.

By using (9), we obtain $\alpha p(z)+\beta \frac{z p^{\prime}(z)}{p(z)}=\alpha \frac{z^{\delta} D R_{\lambda}^{m, n+1} f(z)}{\left(D R_{\lambda}^{m, n} f(z)\right)^{1+\delta}}+$ $+\beta\left[\delta(n+1)-1+(n+2) \frac{D R_{\lambda}^{m, n+2} f(z)}{D R_{\lambda}^{m, n+1} f(z)}-(1+\delta)(n+1) \frac{D R_{\lambda}^{m, n+1} f(z)}{D R_{\lambda}^{m, n} f(z)}\right]$.

By using (7), we have $\alpha p(z)+\frac{\beta z p^{\prime}(z)}{p(z)} \prec \alpha q(z)+\frac{\beta z q^{\prime}(z)}{q(z)}$.

Therefore, the conditions of Lemma 1 are met, so we have $p(z) \prec q(z)$, $z \in U$, i.e. $\frac{z^{\delta} D R_{\lambda}^{m, n+1} f(z)}{\left(D R_{\lambda}^{m, n} f(z)\right)^{1+\delta}} \prec q(z), z \in U$, and $q$ is the best dominant.

Corollary 1. Let $q(z)=\frac{1+A z}{1+B z},-1 \leq B<A \leq 1, m, n \in \mathbb{N}, \lambda \geq 0, z \in U$. Assume that (5) holds. If $f \in \mathcal{A}$ and

$$
\psi_{\lambda}^{m, n}(\alpha, \beta, \delta ; z) \prec \alpha \frac{1+A z}{1+B z}+\beta \frac{(A-B) z}{(1+A z)(1+B z)},
$$

for $\alpha, \beta \in \mathbb{C}, \beta \neq 0,-1 \leq B<A \leq 1,0<\delta \leq 1$ where $\psi_{\lambda}^{m, n}$ is defined in (6), then

$$
\frac{z^{\delta} D R_{\lambda}^{m, n+1} f(z)}{\left(D R_{\lambda}^{m, n} f(z)\right)^{1+\delta}} \prec \frac{1+A z}{1+B z}
$$

and $\frac{1+A z}{1+B z}$ is the best dominant.

Proof. For $q(z)=\frac{1+A z}{1+B z},-1 \leq B<A \leq 1$, in Theorem 1 we get the corollary.

Theorem 2. Let $q$ be convex and univalent in $U$, such that $q(0)=1, m, n \in \mathbb{N}$, $\lambda \geq 0$. Assume that

$$
\operatorname{Re}\left(\frac{\alpha}{\beta} q(z)\right)>0, \text { for } \alpha, \beta \in \mathbb{C}, \beta \neq 0, z \in U .
$$

If $f \in \mathcal{A}, 0<\delta \leq 1, \frac{z^{\delta} D R_{\lambda}^{m, n+1} f(z)}{\left(D R_{\lambda}^{m, n} f(z)\right)^{1+\delta}} \in \mathcal{H}[q(0), 1] \cap Q$ and $\psi_{\lambda}^{m, n}(\alpha, \beta, \delta ; z)$ is univalent in $U$, where $\psi_{\lambda}^{m, n}(\alpha, \beta, \delta ; z)$ is as defined in (6), then

$$
\alpha q(z)+\frac{\beta z q^{\prime}(z)}{q(z)} \prec \psi_{\lambda}^{m, n}(\alpha, \beta, \delta ; z), \quad z \in U,
$$

implies

$$
q(z) \prec \frac{z^{\delta} D R_{\lambda}^{m, n+1} f(z)}{\left(D R_{\lambda}^{m, n} f(z)\right)^{1+\delta}}, \quad z \in U,
$$

and $q$ is the best subordinant. 
ON CERTAIN DIFFERENTIAL SANDWICH THEOREMS INVOLVING A

Proof. Let the function $p$ be defined by $p(z):=\frac{z^{\delta} D R_{\lambda}^{m, n+1} f(z)}{\left(D R_{\lambda}^{m, n} f(z)\right)^{1+\delta}}, z \in U, z \neq 0$, $0<\delta \leq 1, f \in \mathcal{A}$.

By setting $\nu(w):=\alpha w$ and $\phi(w):=\frac{\beta}{w}$ it can be easily verified that $\nu$ is analytic in $\mathbb{C}, \phi$ is analytic in $\mathbb{C} \backslash\{0\}$ and that $\phi(w) \neq 0, w \in \mathbb{C} \backslash\{0\}$.

Since $q$ is convex and univalent function, it follows that $\operatorname{Re}\left(\frac{\nu^{\prime}(q(z))}{\phi(q(z))}\right)=$ $\operatorname{Re}\left(\frac{\alpha}{\beta} q(z)\right)>0$, for $\alpha, \beta \in \mathbb{C}, \beta \neq 0$.

By using (11) we obtain

$$
\alpha q(z)+\beta \frac{z q^{\prime}(z)}{q(z)} \prec \alpha p(z)+\beta \frac{z p^{\prime}(z)}{p(z)} .
$$

Using Lemma 2, we have

$$
q(z) \prec p(z)=\frac{z^{\delta} D R_{\lambda}^{m, n+1} f(z)}{\left(D R_{\lambda}^{m, n} f(z)\right)^{1+\delta}}, \quad z \in U,
$$

and $q$ is the best subordinant.

Corollary 2. Let $q(z)=\frac{1+A z}{1+B z},-1 \leq B<A \leq 1, m, n \in \mathbb{N}, \lambda \geq 0$. Assume that (10) holds. If $f \in \mathcal{A}, \frac{z^{\delta} D R_{\lambda}^{m, n+1} f(z)}{\left(D R_{\lambda}^{m, n} f(z)\right)^{1+\delta}} \in \mathcal{H}[q(0), 1] \cap Q$ and

$$
\alpha \frac{1+A z}{1+B z}+\beta \frac{(A-B) z}{(1+A z)(1+B z)} \prec \psi_{\lambda}^{m, n}(\alpha, \beta, \delta ; z)
$$

for $\alpha, \beta \in \mathbb{C}, \beta \neq 0,-1 \leq B<A \leq 1$, where $\psi_{\lambda}^{m, n}$ is defined in (6), then

$$
\frac{1+A z}{1+B z} \prec \frac{z^{\delta} D R_{\lambda}^{m, n+1} f(z)}{\left(D R_{\lambda}^{m, n} f(z)\right)^{1+\delta}}
$$

and $\frac{1+A z}{1+B z}$ is the best subordinant.

Proof. For $q(z)=\frac{1+A z}{1+B z},-1 \leq B<A \leq 1$ in Theorem 2 we get the corollary.

Combining Theorem 1 and Theorem 2, we state the following sandwich theorem.

Theorem 3. Let $q_{1}$ and $q_{2}$ be analytic and univalent in $U$ such that $q_{1}(z) \neq 0$ and $q_{2}(z) \neq 0$, for all $z \in U$, with $z q_{1}^{\prime}(z)$ and $z q_{2}^{\prime}(z)$ being starlike univalent. Suppose that $q_{1}$ satisfies (5) and $q_{2}$ satisfies (10). If $f \in \mathcal{A}, \frac{z^{\delta} D R_{\lambda}^{m, n+1} f(z)}{\left(D R_{\lambda}^{m, n} f(z)\right)^{1+\delta}} \in$ 
ON CERTAIN DIFFERENTIAL SANDWICH THEOREMS INVOLVING A

$\mathcal{H}[q(0), 1] \cap Q, 0<\delta \leq 1$ and $\psi_{\lambda}^{m, n}(\alpha, \beta, \delta ; z)$ is as defined in (6) univalent in $U$, then

$$
\alpha q_{1}(z)+\frac{\beta z q_{1}^{\prime}(z)}{q_{1}(z)} \prec \psi_{\lambda}^{m, n}(\alpha, \beta, \delta ; z) \prec \alpha q_{2}(z)+\frac{\beta z q_{2}^{\prime}(z)}{q_{2}(z)},
$$

for $\alpha, \beta \in \mathbb{C}, \beta \neq 0$, implies

$$
q_{1}(z) \prec \frac{z^{\delta} D R_{\lambda}^{m, n+1} f(z)}{\left(D R_{\lambda}^{m, n} f(z)\right)^{1+\delta}} \prec q_{2}(z), \quad \delta \in \mathbb{C}, \delta \neq 0,
$$

and $q_{1}$ and $q_{2}$ are respectively the best subordinant and the best dominant.

For $q_{1}(z)=\frac{1+A_{1} z}{1+B_{1} z}, q_{2}(z)=\frac{1+A_{2} z}{1+B_{2} z}$, where $-1 \leq B_{2}<B_{1}<A_{1}<A_{2} \leq 1$, we have the following corollary.

Corollary 3. Let $m, n \in \mathbb{N}, \lambda \geq 0$. Assume that (5) and (10) hold for $q_{1}(z)=$ $\frac{1+A_{1} z}{1+B_{1} z}$ and $q_{2}(z)=\frac{1+A_{2} z}{1+B_{2} z}$, respectively. If $f \in \mathcal{A}, 0<\delta \leq 1, \frac{z^{\delta} D R_{\lambda}^{m, n+1} f(z)}{\left(D R_{\lambda}^{m, n} f(z)\right)^{1+\delta}} \in$ $\mathcal{H}[q(0), 1] \cap Q$ and

$$
\begin{gathered}
\alpha \frac{1+A_{1} z}{1+B_{1} z}+\beta \frac{\left(A_{1}-B_{1}\right) z}{\left(1+A_{1} z\right)\left(1+B_{1} z\right)} \prec \psi_{\lambda}^{m, n}(\alpha, \beta, \delta ; z) \\
\prec \alpha \frac{1+A_{2} z}{1+B_{2} z}+\beta \frac{\left(A_{2}-B_{2}\right) z}{\left(1+A_{2} z\right)\left(1+B_{2} z\right)},
\end{gathered}
$$

for $\alpha, \beta \in \mathbb{C}, \beta \neq 0,-1 \leq B_{2} \leq B_{1}<A_{1} \leq A_{2} \leq 1$, where $\psi_{\lambda}^{m, n}$ is defined in (6), then

$$
\frac{1+A_{1} z}{1+B_{1} z} \prec \frac{z^{\delta} D R_{\lambda}^{m, n+1} f(z)}{\left(D R_{\lambda}^{m, n} f(z)\right)^{1+\delta}} \prec \frac{1+A_{2} z}{1+B_{2} z},
$$

hence $\frac{1+A_{1} z}{1+B_{1} z}$ and $\frac{1+A_{2} z}{1+B_{2} z}$ are the best subordinant and the best dominant, respectively.

Next, our purpose is to find sufficient conditions for certain normalized analytic functions $f$ such that

$q_{1}(z) \prec\left(\frac{a D R_{\lambda}^{m+1, n} f(z)+b D R_{\lambda}^{m, n} f(z)}{(a+b) z}\right)^{\delta} \prec q_{2}(z), \quad z \in U$

where $q_{1}$ and $q_{2}$ are given univalent functions

Theorem 4. Let $\left(\frac{a D R_{\lambda}^{m+1, n} f(z)+b D R_{\lambda}^{m, n} f(z)}{(a+b) z}\right)^{\delta} \in \mathcal{H}(U), f \in \mathcal{A}, z \in U$, $\delta, a, b \in \mathbb{C}, \delta \neq 0, a+b \neq 0, m, n \in \mathbb{N}, \lambda \geq 0$ and let the function $q(z)$ be convex and univalent in $U$ such that $q(0)=1, z \in U$. Assume that

$$
R e\left(1+\frac{\alpha}{\beta} q(z)-\frac{z q^{\prime}(z)}{q(z)}+\frac{z q^{\prime \prime}(z)}{q^{\prime}(z)}\right)>0,
$$


ON CERTAIN DIFFERENTIAL SANDWICH THEOREMS INVOLVING A

for $\alpha, \beta \in \mathbb{C}, \beta \neq 0, z \in U$, and

$$
\begin{gathered}
\psi_{\lambda}^{m, n}(a, b, \alpha, \beta, \delta ; z):=\alpha\left(\frac{a D R_{\lambda}^{m+1, n} f(z)+b D R_{\lambda}^{m, n} f(z)}{(a+b) z}\right)^{\delta}+ \\
\frac{\beta \delta\left[a D R_{\lambda}^{m+2, n} f(z)+(b-a) D R_{\lambda}^{m+1, n} f(z)-b D R_{\lambda}^{m, n} f(z)\right]}{\lambda\left(a D R_{\lambda}^{m+1, n} f(z)+b D R_{\lambda}^{m, n} f(z)\right)} .
\end{gathered}
$$

If $q$ satisfies the following subordination

$$
\psi_{\lambda}^{m, n}(a, b, \alpha, \beta, \delta ; z) \prec \alpha q(z)+\frac{\beta z q^{\prime}(z)}{q(z)},
$$

for $\alpha, \beta \in \mathbb{C}, \beta \neq 0, z \in U$, then

$$
\left(\frac{a D R_{\lambda}^{m+1, n} f(z)+b D R_{\lambda}^{m, n} f(z)}{(a+b) z}\right)^{\delta} \prec q(z), \quad z \in U, \delta \in \mathbb{C}, \delta \neq 0,
$$

and $q$ is the best dominant.

Proof. Let the function $p$ be defined by $p(z):=\left(\frac{a D R_{\lambda}^{m+1, n} f(z)+b D R_{\lambda}^{m, n} f(z)}{(a+b) z}\right)^{\delta}$, $z \in U, z \neq 0, \delta, a, b \in \mathbb{C}, \delta \neq 0, a+b \neq 0, f \in \mathcal{A}$. The function $p$ is analytic in $U$ and $p(0)=1$

Differentiating this function, with respect to $z$, we get

$$
\begin{gathered}
z p^{\prime}(z)=\delta\left(\frac{a D R_{\lambda}^{m+1, n} f(z)+b D R_{\lambda}^{m, n} f(z)}{(a+b) z}\right)^{\delta-1} . \\
\cdot \frac{1}{a+b}\left[\frac{a\left(D R_{\lambda}^{m+1, n} f(z)\right)^{\prime}+b\left(D R_{\lambda}^{m, n} f(z)\right)^{\prime}}{z}-\frac{a D R_{\lambda}^{m+1, n} f(z)+b D R_{\lambda}^{m, n} f(z)}{z^{2}}\right] .
\end{gathered}
$$

We have

$$
\begin{aligned}
& z p^{\prime}(z)=\delta\left(\frac{a D R_{\lambda}^{m+1, n} f(z)+b D R_{\lambda}^{m, n} f(z)}{(a+b) z}\right)^{\delta} \frac{1}{a D R_{\lambda}^{m+1, n} f(z)+b D R_{\lambda}^{m, n} f(z)} . \\
& {\left[a z\left(D R_{\lambda}^{m+1, n} f(z)\right)^{\prime}+b z\left(D R_{\lambda}^{m, n} f(z)\right)^{\prime}-a D R_{\lambda}^{m+1, n} f(z)-b D R_{\lambda}^{m, n} f(z)\right]}
\end{aligned}
$$

By using the identity (3) we obtain

$$
\frac{z p^{\prime}(z)}{p(z)}=\frac{\delta\left[a D R_{\lambda}^{m+2, n} f(z)+(b-a) D R_{\lambda}^{m+1, n} f(z)-b D R_{\lambda}^{m, n} f(z)\right]}{\lambda\left(a D R_{\lambda}^{m+1, n} f(z)+b D R_{\lambda}^{m, n} f(z)\right)}
$$


ON CERTAIN DIFFERENTIAL SANDWICH THEOREMS INVOLVING A

By setting $\theta(w):=\alpha w$ and $\phi(w):=\frac{\beta}{w}, \alpha, \beta \in \mathbb{C}, \beta \neq 0$ it can be easily verified that $\theta$ is analytic in $\mathbb{C}, \phi$ is analytic in $\mathbb{C} \backslash\{0\}$ and that $\phi(w) \neq 0$, $w \in \mathbb{C} \backslash\{0\}$.

Also, by letting $Q(z)=z q^{\prime}(z) \phi(q(z))=\frac{\beta z q^{\prime}(z)}{q(z)}$,we find that $Q(z)$ is starlike univalent in $U$.

Let $h(z)=\theta(q(z))+Q(z)=\alpha q(z)+\frac{\beta z q^{\prime}(z)}{q(z)}, z \in U$

If we derive the function $Q$, with respect to $z$, perform calculations, we have $\operatorname{Re}\left(\frac{z h^{\prime}(z)}{Q(z)}\right)=\operatorname{Re}\left(1+\frac{\alpha}{\beta} q(z)-\frac{z q^{\prime}(z)}{q(z)}+\frac{z q^{\prime \prime}(z)}{q^{\prime}(z)}\right)>0$.

By using (9), we obtain

$$
\begin{gathered}
\alpha p(z)+\beta \frac{z p^{\prime}(z)}{p(z)}=\alpha\left(\frac{a D R_{\lambda}^{m+1, n} f(z)+b D R_{\lambda}^{m, n} f(z)}{(a+b) z}\right)^{\delta}+ \\
+\frac{\beta \delta\left[a D R_{\lambda}^{m+2, n} f(z)+(b-a) D R_{\lambda}^{m+1, n} f(z)-b D R_{\lambda}^{m, n} f(z)\right]}{\lambda\left(a D R_{\lambda}^{m+1, n} f(z)+b D R_{\lambda}^{m, n} f(z)\right)} .
\end{gathered}
$$

By using (15), we have $\alpha p(z)+\beta \frac{z p^{\prime}(z)}{p(z)} \prec \alpha q(z)+\beta \frac{z q^{\prime}(z)}{p(z)}$.

From Lemma 1, we have $p(z) \prec q(z), z \in U$, i.e.,

$$
\left(\frac{a D R_{\lambda}^{m+1, n} f(z)+b D R_{\lambda}^{m, n} f(z)}{(a+b) z}\right)^{\delta} \prec q(z),
$$

$z \in U, \delta \in \mathbb{C}, \delta \neq 0$, and $q$ is the best dominant.

Corollary 4. Let $q(z)=\frac{1+A z}{1+B z}, z \in U,-1 \leq B<A \leq 1, m, n \in \mathbb{N}, \lambda \geq 0$. Assume that (13) holds. If $f \in \mathcal{A}$ and

$$
\psi_{\lambda}^{m, n}(a, b, \alpha, \beta, \delta ; z) \prec \alpha \frac{1+A z}{1+B z}+\beta \frac{(A-B) z}{(1+A z)(1+B z)},
$$

for $\alpha, \beta \in \mathbb{C}, \beta \neq 0,-1 \leq B<A \leq 1$, where $\psi_{\lambda}^{m, n}$ is defined in (14), then

$$
\left(\frac{a D R_{\lambda}^{m+1, n} f(z)+b D R_{\lambda}^{m, n} f(z)}{(a+b) z}\right)^{\delta} \prec \frac{1+A z}{1+B z}, \quad \delta \in \mathbb{C}, \delta \neq 0,
$$

and $\frac{1+A z}{1+B z}$ is the best dominant.

Proof. For $q(z)=\frac{1+A z}{1+B z},-1 \leq B<A \leq 1$, in Theorem 4 we get the corollary. 
ON CERTAIN DIFFERENTIAL SANDWICH THEOREMS INVOLVING A

Theorem 5. Let $q$ be convex and univalent in $U$ such that $q(0)=1$. Assume that

$$
\operatorname{Re}\left(\frac{\alpha}{\beta} q(z)\right)>0, \text { for } \alpha, \beta \in \mathbb{C}, \beta \neq 0 .
$$

If $f \in \mathcal{A}, \delta, a, b \in \mathbb{C}, \delta \neq 0, a+b \neq 0,\left(\frac{a D R_{\lambda}^{m+1, n} f(z)+b D R_{\lambda}^{m, n} f(z)}{(a+b) z}\right)^{\delta} \in$ $\mathcal{H}[q(0), 1] \cap Q$ and

$\psi_{\lambda}^{m, n}(a, b, \alpha, \beta, \delta ; z)$ is univalent in $U$, where $\psi_{\lambda}^{m, n}(a, b, \alpha, \beta, \delta ; z)$ is as defined in (14), then

$$
\alpha q(z)+\frac{\beta z q^{\prime}(z)}{q(z)} \prec \psi_{\lambda}^{m, n}(a, b, \alpha, \beta, \delta ; z)
$$

implies

$$
q(z) \prec\left(\frac{a D R_{\lambda}^{m+1, n} f(z)+b D R_{\lambda}^{m, n} f(z)}{(a+b) z}\right)^{\delta}, \quad \delta \in \mathbb{C}, \delta \neq 0, z \in U,
$$

and $q$ is the best subordinant.

Proof. Let the function $p$ be defined by $p(z):=\left(\frac{a D R_{\lambda}^{m+1, n} f(z)+b D R_{\lambda}^{m, n} f(z)}{(a+b) z}\right)^{\delta}$, $z \in U, z \neq 0, a, b \in \mathbb{C}, a+b \neq 0, \delta \in \mathbb{C}, \delta \neq 0, f \in \mathcal{A}$. The function $p$ is analytic in $U$ and $p(0)=1$.

By setting $\nu(w):=\alpha w$ and $\phi(w):=\frac{\beta}{w}$ it can be easily verified that $\nu$ is analytic in $\mathbb{C}, \phi$ is analytic in $\mathbb{C} \backslash\{0\}$ and that $\phi(w) \neq 0, w \in \mathbb{C} \backslash\{0\}$.

Since $q$ is convex and univalent function, it follows that $\operatorname{Re}\left(\frac{\nu^{\prime}(q(z))}{\phi(q(z))}\right)=$ $\operatorname{Re}\left(\frac{\alpha}{\beta} q(z)\right)>0$, for $\alpha, \beta \in \mathbb{C}, \beta \neq 0$.

By using (11) we obtain

$$
\alpha q(z)+\beta \frac{z q^{\prime}(z)}{q(z)} \prec \alpha p(z)+\beta \frac{z p^{\prime}(z)}{p(z)} .
$$

From Lemma 2, we have

$$
q(z) \prec p(z)=\left(\frac{a D R_{\lambda}^{m+1, n} f(z)+b D R_{\lambda}^{m, n} f(z)}{(a+b) z}\right)^{\delta}, \quad z \in U, \delta \in \mathbb{C}, \delta \neq 0,
$$

and $q$ is the best subordinant.

Corollary 5. Let $q(z)=\frac{1+A z}{1+B z},-1 \leq B<A \leq 1, z \in U, m, n \in \mathbb{N}$, $\lambda \geq 0$. Assume that (19) holds. If $f \in \mathcal{A},\left(\frac{a D R_{\lambda}^{m+1, n} f(z)+b D R_{\lambda}^{m, n} f(z)}{(a+b) z}\right)^{\delta} \in$ 
ON CERTAIN DIFFERENTIAL SANDWICH THEOREMS INVOLVING A

$\mathcal{H}[q(0), 1] \cap Q, \delta \in \mathbb{C}, \delta \neq 0$ and

$$
\alpha \frac{1+A z}{1+B z}+\beta \frac{(A-B) z}{(1+A z)(1+B z)} \prec \psi_{\lambda}^{m, n}(a, b, \alpha, \beta, \delta ; z),
$$

for $\alpha, \beta \in \mathbb{C}, \beta \neq 0,-1 \leq B<A \leq 1$, where $\psi_{\lambda}^{m, n}$ is defined in (14), then

$$
\frac{1+A z}{1+B z} \prec\left(\frac{a D R_{\lambda}^{m+1, n} f(z)+b D R_{\lambda}^{m, n} f(z)}{(a+b) z}\right)^{\delta}, \delta \in \mathbb{C}, \delta \neq 0, a, b \in \mathbb{C}, a+b \neq 0
$$

and $\frac{1+A z}{1+B z}$ is the best subordinant.

Proof. For $q(z)=\frac{1+A z}{1+B z},-1 \leq B<A \leq 1$, in Theorem 5 we get the corollary.

Combining Theorem 4 and Theorem 5, we state the following sandwich theorem.

Theorem 6. Let $q_{1}$ and $q_{2}$ be convex and univalent in $U$ such that $q_{1}(z) \neq 0$ and $q_{2}(z) \neq 0$, for all $z \in U$. Suppose that $q_{1}$ satisfies (13) and $q_{2}$ satisfies (19).

If $f \in \mathcal{A},\left(\frac{a D R_{\lambda}^{m+1, n} f(z)+b D R_{\lambda}^{m, n} f(z)}{(a+b) z}\right)^{\delta} \in \mathcal{H}[q(0), 1] \cap Q, \delta \in \mathbb{C}, \delta \neq 0$, $a, b \in \mathbb{C}, a+b \neq 0$ and $\psi_{\lambda}^{m, n}(a, b, \alpha, \beta, \delta ; z)$ is as defined in (14) univalent in $U$, then

$$
\alpha q_{1}(z)+\frac{\beta z q_{1}^{\prime}(z)}{q_{1}(z)} \prec \psi_{\lambda}^{m, n}(a, b, \alpha, \beta, \delta ; z) \prec \alpha q_{2}(z)+\frac{\beta z q_{2}^{\prime}(z)}{q_{2}(z)},
$$

for $\alpha, \beta \in \mathbb{C}, \beta \neq 0$, implies

$q_{1}(z) \prec\left(\frac{a D R_{\lambda}^{m+1, n} f(z)+b D R_{\lambda}^{m, n} f(z)}{(a+b) z}\right)^{\delta} \prec q_{2}(z), \quad z \in U, \delta \in \mathbb{C}, \delta \neq 0$,

and $q_{1}$ and $q_{2}$ are respectively the best subordinant and the best dominant.

For $q_{1}(z)=\frac{1+A_{1} z}{1+B_{1} z}, q_{2}(z)=\frac{1+A_{2} z}{1+B_{2} z}$, where $-1 \leq B_{2}<B_{1}<A_{1}<A_{2} \leq 1$, we have the following corollary.

Corollary 6. Let $m, n \in \mathbb{N}, \lambda \geq 0$. Assume that (13) and (19) hold for $q_{1}(z)=\frac{1+A_{1} z}{1+B_{1} z}$ and $q_{2}(z)=\frac{1+A_{2} z}{1+B_{2} z}$, respectively. If $f \in \mathcal{A},\left(\frac{D R_{\lambda}^{m+1, n} f(z)}{D R_{\lambda}^{m, n} f(z)}\right)^{\delta} \in$ $\mathcal{H}[q(0), 1] \cap Q$ and

$$
\alpha \frac{1+A_{1} z}{1+B_{1} z}+\beta \frac{\left(A_{1}-B_{1}\right) z}{\left(1+A_{1} z\right)\left(1+B_{1} z\right)} \prec \psi_{\lambda}^{m, n}(a, b, \alpha, \beta, \delta ; z)
$$


ON CERTAIN DIFFERENTIAL SANDWICH THEOREMS INVOLVING A

$$
\prec \alpha \frac{1+A_{2} z}{1+B_{2} z}+\beta \frac{\left(A_{2}-B_{2}\right) z}{\left(1+A_{2} z\right)\left(1+B_{2} z\right)},
$$

for $\alpha, \beta \in \mathbb{C}, \beta \neq 0,-1 \leq B_{2} \leq B_{1}<A_{1} \leq A_{2} \leq 1$, where $\psi_{\lambda}^{m, n}$ is defined in (14), then

$$
\begin{array}{r}
\frac{1+A_{1} z}{1+B_{1} z} \prec\left(\frac{a D R_{\lambda}^{m+1, n} f(z)+b D R_{\lambda}^{m, n} f(z)}{(a+b) z}\right)^{\delta} \prec \frac{1+A_{2} z}{1+B_{2} z}, \\
z \in U, \delta \in \mathbb{C}, \delta \neq 0, a, b \in \mathbb{C}, a+b \neq 0
\end{array}
$$

hence $\frac{1+A_{1} z}{1+B_{1} z}$ and $\frac{1+A_{2} z}{1+B_{2} z}$ are the best subordinant and the best dominant, respectively.

Acknowledgement. This work was partially suported by the strategic project POSDRU/159/1.5/S/138963 - "Perform".

\section{References}

[1] A. Alb Lupas, Certain differential subordinations using Sălăgean and Ruscheweyh operators, Acta Universitatis Apulensis, No. 29/2012, 125129.

[2] A. Alb Lupas, A note on differential subordinations using Sălăgean and Ruscheweyh operators, Romai Journal, vol. 6, nr. 1(2010), 1-4.

[3] A. Alb Lupas, Certain differential superordinations using Sălăgean and Ruscheweyh operators, Analele Universităţii din Oradea, Fascicola Matematica, Tom XVII, Issue no. 2, 2010, 209-216.

[4] A. Alb Lupas, Certain differential subordinations using a generalized Sălăgean operator and Ruscheweyh operator I, Journal of Mathematics and Applications, No. 33 (2010), 67-72.

[5] A. Alb Lupas, Certain differential subordinations using a generalized Sălăgean operator and Ruscheweyh operator II, Fractional Calculus and Applied Analysis, Vol 13, No. 4 (2010), 355-360.

[6] A. Alb Lupas, Certain differential superordinations using a generalized Sălăgean and Ruscheweyh operators, Acta Universitatis Apulensis nr. 25/2011, 31-40.

[7] F.M. Al-Oboudi, On univalent functions defined by a generalized Sălăgean operator, Ind. J. Math. Math. Sci., 27 (2004), 1429-1436. 
ON CERTAIN DIFFERENTIAL SANDWICH THEOREMS INVOLVING A

[8] L. Andrei, Differential subordination results using a generalized Sălăgean operator and Ruscheweyh operator, Acta Universitatis Apulensis (to appear).

[9] L. Andrei, Some differential subordination results using a generalized Sălăgean operator and Ruscheweyh operator, submitted.

[10] L. Andrei, Differential superordination results using a generalized Sălăgean operator and Ruscheweyh operator, Analele Universitatii Oradea (to appear).

[11] L. Andrei, Some differential superordination results using a generalized Sălăgean operator and Ruscheweyh operator, submitted.

[12] L. Andrei, Differential Sandwich Theorems using a generalized Sălăgean operator and Ruscheweyh operator, submitted.

[13] L. Andrei, On some differential sandwich theorems using a generalized Sălăgean operator and Ruscheweyh operator, Journal of Computational Analysis and Applications (to appear)

[14] T. Bulboacă, Classes of first order differential superordinations, Demonstratio Math., Vol. 35, No. 2, 287-292.

[15] M. Darus, K. Al-Shaqsi, Differential sandwich theorems with generalised derivative operator, Advanced Technologies, October, Kankesu Jayanthakumaran (Ed), ISBN:978-953-307-009-4 2009

[16] R.W. Ibrahim, M. Darus, On sandwich theorems of analytic functions involving Noor integral operator, African Journal of Mathematics and Computer Science Research, vol. 2, pp.132-137, 2009

[17] S.S. Miller, P.T. Mocanu, Subordinants of Differential Superordinations, Complex Variables, vol. 48, no. 10, 815-826, October, 2003.

[18] S.S. Miller, P.T. Mocanu, Differential Subordinations: Theory and Applications, Marcel Dekker Inc., New York, 2000.

[19] St. Ruscheweyh, New criteria for univalent functions, Proc. Amet. Math. Soc., 49 (1975), 109-115.

[20] G. St. Sălăgean, Subclasses of univalent functions, Lecture Notes in Math., Springer Verlag, Berlin, 1013 (1983), 362-372.

[21] T.N. Shanmugan, M.P.Jeyaraman, On sandwich theorems for certain subclasses of analytic functions associated with Dziok- Srivastava operator, Taiwanese Journal of Mathematics, vol. 13, no.6B, 1949-1961, 2009 
ON CERTAIN DIFFERENTIAL SANDWICH THEOREMS INVOLVING A

[22] T.N. Shanmugan, C. Ramachandran, M. Darus, S. Sivasubramanian, Differential sandwich theorems for some subclasses of analytic functions involving a linear operator, Acta Math. Univ. Comenianae, 16 (2007), no. $2,287-294$.

[23] T.N. Shanmugan, S. Srikandan, B.A. Frasin, S.Kavitha, On sandwich theorems for certain subclasses of analytic functions involving CarlsonShaffer operator, J. Korean Math. Soc. 45, no.3, pp.611-620, 2008

Loriana ANDREI,

Department of Mathematics and Computer Science,

University of Oradea,

1 Universitatii Street, 410087, Oradea, Romania.

Department of Mathematics,

University of Pitesti,

Targul din Vale Street, No. 1, 110040, Pitesti, Romania

Email: lori_andrei@yahoo.com 
ON CERTAIN DIFFERENTIAL SANDWICH THEOREMS INVOLVING A GENERALIZED SALAGEAN OPERATOR AND RUSCHEWEYH OPERATOR 